\title{
God, Logic, and Quantum Information
}

\author{
Vasil Penchev, vasildinev@gmail.com \\ Bulgarian Academy of Sciences: Institute of Philosophy and Sociology: \\ Dept. of Logical Systems and Models
}

\begin{abstract}
Quantum information is discussed as the universal substance of the world. It is interpreted as that generalization of classical information, which includes both finite and transfinite ordinal numbers. On the other hand, any wave function and thus any state of any quantum system is just one value of quantum information. Information and its generalization as quantum information are considered as quantities of elementary choices. Their units are correspondingly a bit and a qubit. The course of time is what generates choices by itself, thus quantum information and any item in the world in final analysis. The course of time generates necessarily choices so: The future is absolutely unorderable in principle while the past is always well-ordered and thus unchangeable. The present as the mediation between them needs the well-ordered theorem equivalent to the axiom of choice. The latter guarantees the choice even among the elements of an infinite set, which is the case of quantum information. The concrete and abstract objects share information as their common base, which is quantum as to the formers and classical as to the latters. The general quantities of matter in physics, mass and energy can be considered as particular cases of quantum information. The link between choice and abstraction in set theory allows of "Hume's principle" to be interpreted in terms of quantum mechanics as equivalence of "many" and "much" underlying quantum information. Quantum information as the universal substance of the world calls for the unity of physics and mathematics rather than that of the concrete and abstract objects and thus for a form of quantum neo-Pythagoreanism in final analysis.
\end{abstract}

Key words: God, logic, information, quantum information, choice, axiom of choice, matter, mass, energy 


\section{Introduction}

The concept of matter in physics can be considered as a generalized form of information, that of quantum information involved by quantum mechanics. Furthermore, the concept of information can unify the concrete and abstract objects while the notions of matter and energy in physics demark them. Thus, information can be seen as the universal substance of the world and therefore, as the relevant generalization of the notions of mass and energy in physics referring only to the world of the concrete objects.

The concept of quantum information introduced by quantum mechanics allows of an interpretation of the world as conscious, and of logic as the result of the action of that conscious medium.

As usual, quantum mechanics and the theory of quantum information call that interpretation "quantum computer" or "the universe (world) as a quantum computer". However, one can show that this "quantum computer" possesses the capability of free choice and some kind of natural teleology. The link between the former and latter generates one phenomenon, which can be investigated by science: It can be called the free will of the universe and interpreted as a scientific conception of God or as a hypostasis of Him studied by theology.

The same viewpoint includes all logics or the conception of universal logics in a natural way. Any logic "of anything" can be seen as a partial ordering and thus as a stage of the universal and single wellordering of the universe going to the past accomplished by the "universe as a quantum computer". Thus any that should be a partial result in the ordering in the course of time from future to past by the meditation of the present and of the choices made in the present.

\section{Arguments:}

(I) The course of time can be described in terms of quantum mechanics as follows: The absolutely coherent state of the future de-coheres gradually into less and less entangled quantum systems by means of choices (or "measurements") made in the present. Thus those entangled quantum systems are being transformed in mechanical systems absolutely separated to each other in and after the limit of the past.

(II) Universal logic can be considered as the series of partial orderings of some universal class, e.g. that of all sets. Then any given logic will be exactly one member of that series and can be defined (1) by the set, to which the partial orderings refer, and (2) by the rule, which can generate just the partial ordering, i.e. by the property, which describes the set of all well-orderings representing the partial ordering in question. The definition (1) determines the logic as the "logic of something" where that "something" is the set, which has to be ordered and its "logic" means the way and degree of the ordering. The definition (1) includes both (1.1) any scientific theory as the logic of the object of the theory, and properly (1.2) the "logics of something" where that "something" is some set more interesting by the rule (2), which can generate rather than by itself. The definition (2) includes both (2.1) the case of the explicit property generating (all) well-orderings on any set independently of the interpretation of its elements and (2.2) the "topological representation" of the logic as the description of all well-orderings one by one rather than a common property determining unambiguously all well-orderings as it is in the former case.

(III) The collaboration of quantum mechanics by the conception of quantum information allows of a natural ontological interpretation of universal logic. There is a natural process of ordering in the course of time independent of what is ordered. What is ordered can be e.g. the world, i.e. the universe as a whole, or any part of it, i.e. any quantum system. So, universal logic can be interpreted as the successive partial results in the process of ordering independent of what is ordered. That ordering is a well-ordering and it originates from the course of time. According to quantum mechanics, the general course of time can be described as a (well-) ordering in thus: The coherent state of future is being ordered into the single well- 
ordering of past here by the meditation of the all choices in present accomplishing the ordering. That universal well-ordering in turn orders well all partial results, each of which is some logic. Consequently, the series of all logics turns out to be well-ordered if that is the conception of universal logic. The distance between any two logics can be measured by the quantity of (quantum) information. Any logic is unambiguously determined by the information distance from the coherent state (the "absolute future"), on the one hand, and from the single well-ordering (the "absolute past").

\section{A few preliminary notes}

The first one refers to quantum information:

The conception of quantum information was introduced in the theory of quantum information studying the phenomena of entanglement in quantum mechanics: The entanglement was theoretically forecast in the famous papers of Einstein, Podolsky, and Rosen (1935: 777) and independently by Schrödinger (1935: 807) deducing it from Hilbert space, on which is based the mathematical formalism of quantum mechanics. However, the former three demonstrated the forecast phenomenon as the proof of the alleged "incompleteness of quantum mechanics".

John Bell (1964: 195) deduced a sufficient condition as an experimentally verifiable criterion in order to distinguish classical from quantum correlation (entanglement). Aspect, Grangier, and Roger (1981: 460; 1982: 91) confirmed experimentally the existence of quantum correlations exceeding the upper limit of all possible classical correlations. The theory of quantum information has thrived since the end of the last century in the areas of quantum computer, quantum communication, and quantum cryptography.

Quantum information can be considered as a quantity measured in qubits: The notion of quantum bit (or 'qubit') underlies that of quantum information:

'Qubit' is: $\alpha|\mathbf{0}\rangle+\beta|\mathbf{1}\rangle$ where $\alpha, \beta$ are two complex numbers such that $|\alpha|^{2}+|\beta|^{2}=1$, and $|\mathbf{0}\rangle,|\mathbf{1}\rangle$ are any two orthonormal vectors (e.g. the orthonormal bases of any two subspaces) in any vector space (e.g. Hilbert space, Euclidean space, etc.).

A qubit is isomorphic to a unit ball under the following conditions: A qubit is equivalently representable as a unit ball in Euclidean space and two points, the one chosen within the ball, and the other being the orthogonal projection on its surface.

Consequently, the qubit links the Hilbert space of quantum mechanics to the Minkowski space of special relativity and even to the pseudo-Riemannian space of general relativity (the latter by the additional mediation of the concept of entanglement). The "Banach-Tarski paradox" (Banach, Tarski 1924: 244) connects the axiom of choice and the unit-ball representation of a qubit.

Hilbert space can be represented as a "tape" of qubits:

Given any point in the complex Hilbert space as a vector $\left\{C_{1}, C_{2}, \ldots C_{n}, C_{n+1}, \ldots\right\}$, one can replace any successive couple of its components such as $\left(\left\{C_{1}, C_{2}\right\},\left\{C_{2}, C_{3}\right\}, \ldots\left\{C_{n-1}, C_{n}\right\} \ldots\right)$ with a single corresponding qubit $\left\{Q_{1}, Q_{2}, \ldots, Q_{n}, Q_{n+1}, \ldots\right\}$ such that:

$$
\alpha_{n}=\frac{C_{n}}{(+) \sqrt{\left|C_{n}\right|^{2}+\left|C_{n+1}\right|^{2}}} ; \beta_{n}=\frac{C_{n+1}}{(+) \sqrt{\left|C_{n}\right|^{2}+\left|C_{n+1}\right|^{2}}}
$$

if $C_{n}, C_{n+1}$ are not both 0 . However if both are 0 one needs to add conventionally the center $\left(\alpha_{n}=0, \beta_{n}=0\right)$ to conserve the mapping of Hilbert space and an infinite qubit tape to be one-to-one.

A bit and a qubit can be compared: Then if any bit is an elementary binary choice between two disjunctive options usually designated by " 0 " and " 1 ", any qubit is a choice between a continuum of disjunctive 
options as many (or "much") as the points of the surface of the unit ball. Thus the concept of choice is the core of computation and information. It is what can unify the classical and quantum case, and the demarcation between them is the bound between a finite vs. infinite number of the alternatives of the corresponding choice.

A Turing machine can be juxtaposed with a quantum Turing machine: The quantum Turing machine processes quantum information correspondingly qubit by qubit serially, but in parallel within any qubit: The axiom of choice formalizes that parallel processing as the choice of the result. Even the operations on a qubit can be the same as on a bit. The only difference should be for the command "write/ read": It should be a value of either a binary (finite) or an infinite set.

Quantum information can be considered as the information of an infinite set as an ordinal and as complexity: The quantum information introduced by quantum mechanics is equivalent to that generalization of the classical information from finite to infinite series or collections. Indeed information can be interpreted as the number of choices necessary to be reached an ordering of some item from another ordering of the same item or from the absence of ordering. Then the quantity of information is the quantity of choices measured in the units of elementary choice. The quantity of quantum information is the ordinal corresponding to the infinite series in question.

The second preliminary note refers to the conception of "ordinal number" in set theory and its application in quantum mechanics. There are two well-known common definitions of 'ordinal': Both definitions of 'ordinal number' are interpretable in terms of quantum mechanics:

The Cantor - Russell definition (Cantor 1897: 207; Whitehead, Russell 1927: 18) is admissible as the ordinals are small: " $\omega$ " is an enough limit. The ordinal defined in Cantor - Russell generates a statistical ensemble while that in Neumann (1923: 199), a well-ordering. Both correspond one-to-one to a coherent state as the one and same quantity of quantum information containing in it.

The interpretation of the two "kinds" of ordinal numbers in terms of quantum mechanics is the following: The relation between the statistical ensemble and the single and unknown well-ordering is the relation between an ordinal defined correspondingly in Cantor - Russell or in Neumann. The ordinal defined in Neumann should be interpreted as a representative of 'determinism' for any statistical ensemble corresponding one-to-one to an ordinal defined in Cantor - Russell. However, this representative exists only "purely" for it is a mapping of a coherent state necessarily requiring the axiom of choice.

The third preliminary note concerns the concept of the "length of now" after de Broglie. The "length of now" of any quantum entity can be defined as the period of the de Broglie wave (De Broglie 1925: 22), which can be associated to that quantum entity: Thus the "length of now" should be reciprocal to the energy (mass) of the quantum entity: Then the "length of now" of the device should be a randomly chosen point from the segment of the "length of now" of the quantum entity therefore including the future and the past of the apparatus uniformly.

\section{Mass, energy and information as linked physical quantities}

Contemporary physics introduces the notion of matter and quantity of mass as a form of energy according to Einstein's famous equation $E=\mathrm{mc}^{2}$. The physical world and all entities within it (the concrete objects) share that quantity of matter. However, there exist abstract objects, which do not belong to the physical world. Thus, the physical concept of mass does not refer to them. Consequently, that quantity of mass is the demarcation between those two worlds: that of the concrete objects and that of the abstract ones. Any entity should belong either to the one or to the other. 
All abstract objects share a common quantity, that of information. It can be defined in different ways, partly equivalent to each other. It can be interpreted also as the complexity of a given abstract object, e.g. as the length of the shortest algorithm (or the number of the corresponding Turing machine), by which the object at issue can be constructed (Kolmogorov 1968: 662).

The dimensionless physical quantity of thermodynamic entropy shares the same or similar mathematical formula as information. However, it always refers to some statistical ensembles of material (energetic) entities and thus the demarcation between mass (energy) and information is conserved though the concept of information unifies both concrete and abstract objects. Information in both cases can be considered as a quantity describing the degree of ordering (or disordering, or complexity) of any collection either of abstract or of concrete objects

Furthermore, any physical entity shares quantum information. The concept of quantum information introduced by quantum mechanics allows even more: Any physical entity to be interpreted as some nonzero quantity of quantum information, which can be seen as that generalization of information, which is relevant to infinite collections for the classically defined information can refer only to finite ones.

Then the following hypothesis can be offered:

The quantities of mass and energy are interpretable as some nonzero amount of quantum information. Thus the demarcation between the concrete and abstract objects can be understood as the boundary between infinity and finiteness in a rigorous and even mathematical sense. This allows of diffusing concepts between philosophy of mathematics and quantum mechanics, on the one hand, and ontology, on the other hand.

Mass, energy, and matter can be considered as forms of information. The core is the following: the physical concepts of mass, energy and matter are interpreted as the notion of information in the case of quantum information, i.e. as the information in an infinite collection. Furthermore, the mathematical analysis of the relation between infinity and finiteness can be transferred to elucidate the essence of matter even in an ontological sense.

Mass and energy can be referred to the complexity of infinite sets. Energy (and therefore mass) can be interpreted as the change of the complexity of a relevant infinite set in thus: Energy is the change of that transfinite ordinal representing the complexity per a unit of transfinite well-ordering. That unit of the number of sells necessary for that transfinite well-ordering should be a unit of time. The change of the transfinite ordinal number should be the corresponding change of probability being due to the change of a wave function.

\section{Choice and information}

Choice should be put in the base of information. The notion of choice grounds that of information. The latter can be seen as the quantity of elementary choices in units of choice, which are also units of information. The generalization of information through the boundary of infinity as quantum information requires the axiom of choice (Zermelo 1904: 514) to legitimate the notion of choice as to infinity.

The axiom of choice applied to quantum mechanics implies quantum invariance in relation to the choice in the following sense: A few theorems (Neumann 1932: 167; Kochen, Specker 1968: 59) deduce from the mathematical formalism of Hilbert space that no hidden variable and thus no well-ordering is allowed for any coherent state in quantum mechanics. However, the latter is well-ordered after measurement and thus needs the well-ordering theorem equivalent to the axiom of choice. The epistemological equivalence of a quantum system before and after measurement forces the invariance to 
the axiom of choice. That invariance is shared by the Hilbert space formalism. This fact can be called quantum invariance as to quantum mechanics.

Choice can be generalized to infinity: One can demonstrate that quantum mechanics involves and even develops implicitly the concept of choice as to infinity, on the one hand, and set theory (the so-called paradox of Skolem (1970: 138) based on the axiom of choice) does the same, on the other hand. Thus the understanding of matter as information elucidates how choice underlies matter and even ontology at all. The concept of quantum information can be introduced in different ways:

One of them defines it by means of Hilbert space and thus any point in it, which is equivalent to a wave function, i.e. to a state of some quantum system, can be considered as a certain value of the quantity of quantum information.

A Turing machine can refer to a quantum computer under certain conditions: That visualization allows of highlighting the fundamental difference between the Turing machine (Turing 1937: 230) and quantum computer (Deutsch 1985: 97; Deutsch 1989: 73; Yao 1993: 352): The choice of an element of an uncountable set requires the axiom of choice necessarily. The axiom of choice being non-constructive is the relevant reference frame to the concept of quantum algorithm: The latter in turn involves a constructive process of solving or computation having an infinite and even uncountable number of steps therefor.

Information can be defined as the number of primary choices: The concept of information can be interpreted as the quantity of the number of primary choices. Furthermore, the Turing machine either classical or quantum as a model links computation to information directly: The quantity of information can be thought as the sum of the change bit by bit or qubit by qubit, i.e. as the change of a number written either by two or by infinitely many digits.

The following equating should hold:

A cell of a (quantum) Turing tape $=$ a qubit $=$ a choice of (quantum) information $=$ an "infinite digit"

The "leap" from information to quantum information is through the boundary of infinity: The generalization from information to quantum information can be interpreted as the corresponding generalization of 'choice': from the choice between two (or any finite number of) disjunctive alternatives to infinitely many (and even "much") alternatives. Thus the distinction between the classical and quantum case can be limited within any cell of an algorithm or (qu)bit of information.

\section{Abstraction and choice}

Abstraction and choice are implicitly defined in set theory: The link between abstraction and choice in the foundation of set theory can distinguish unambiguously the "good" principles of abstraction from the "bad" ones. The good abstraction is always a choice in the sense of set theory; or in other words, that abstraction, to which a choice does not correspond, is a "bad abstraction" implying contradictions.

The abstraction as a generalization can be compared with the choice by means of two examples: Abstraction was initially allowed to be unrestricted in "naïve set theory" therefore admitting a lot of paradoxes. Zermelo (Zermelo 1908: 261) was who offered the relevant out way restricting the abstraction in set theory in fact by means of choice: a set is not only the abstraction of its elements, but also it can be chosen from another set.

Linnebo's concept of "COLAPSE" (Linnebo 2010: 144) and Popper's principle of falsifiability (Popper 1935: 13) are two possible examples more of the complement of the generalization by the relevant choice of the abstracted. 
The axiom of choice can be referred to the axiom scheme of specification: The concepts of abstraction or that of choice in set theory is fundamental (like that of point in geometry) and cannot be defined rigorously otherwise than contextually and indirectly by the axioms in set theory: As the axiom of choice can correspond to 'choice' as the axiom scheme of specification, to 'abstraction'. Their intuitions are the opportunities accordingly an element to be chosen from a set or all elements of a set to be specified by a single logical function.

A few words can be said about the logical equivalence of choice and abstraction: One can designate as the "name" or "natural name" of a set that logical function, which is equivalent to it according to the corresponding axiom (or axiom scheme) of abstraction in set theory: Then, what is the relation between the name and the choice of one and the same set? Can a set be chosen without having any name? Or vice versa: can a set be named without being chosen? One can suggest the equivalence of the name and the choice of one and the same set for it seems intuitively justified.

An example can be given by the "Gödel first incompleteness theorem": Furthermore, "This set has this name" should be a decidable proposition. However, the so-called Gödel first incompleteness theorem, "Satz VI" (Gödel 1931: 187) implies that there are such sets and such names, about which that proposition is not decidable if the conditions of the validity of the theorem are satisfied. This implies for the name of any set to be imposed suitable restrictions, which should exclude the application of Gödel's theorem: One can choose as a name any proposition out of its conditions.

One believes that this can be avoided by the restriction in the corresponding postulate in set theory for the names to be finite or to consist of a finite set of free variables. However, what about the sets having no finite name, but possessing an infinite name? Is there at least one set of that kind? Obviously, yes, there is: e.g. any transcendental number without any special designation like " $\pi$ ", "e", etc. One need an actual infinite set, e.g. that of its digits, in order to construct its name.

However, the restriction of name in the corresponding axiom scheme in set theory about abstraction should exclude it thus saving the theory from the Gödel undecidable propositions as names of sets The axiom of choice would distinguish unambiguously even between them: The transcendental number being single can be chosen while any set specified by some undecidable proposition cannot be chosen.

Furthermore, abstraction and choice can be defined in terms of quantum mechanics, too: 'Choice' is then the relation of a coherent state (or superposition) and a measured value of it (or an element of the corresponding statistical ensemble).

The reverse relation (either of a single element or of the whole statistical ensemble) to the coherent state can be accordingly interpreted as that 'abstraction' in terms of quantum mechanics.

Both abstraction and well-ordering may be referred to quantum mechanics as coherence and decoherence: Any well-ordering can be considered as an ordered series of choices: Thus a mapping of a coherent state into a statistical ensemble can be interpreted in terms both of transfinite ordinals and wave functions as the quantity of quantum information containing in it. Furthermore, the quantity of quantum information should be invariant both to abstraction and to choice (as they are defined in quantum mechanics above) after the wave functions (points in Hilbert space) and the transfinite ordinals are mapped one-to-one into each other.

"Hume's principle" is introduced as a fundamental logical principle: In fact the so-called principle of Hume is suggested by a contemporary logician, George Boolos (1987: 3). Its sense seems quite simple and obvious: The enumeration does not change the number of the enumerated items whatever they are. The enumeration cannot change information. Thus the number or information should be invariant to whether the objects are abstract or concrete. 
Or in other words, any number is the abstraction of all sets having the same number of elements, whatever these elements or sets are.

"Hume's principle" generalized in terms of quantum mechanics should be sound thus: In the quantum principle of Hume " $G_{\mathrm{s}}$ " should be interpreted as some "many" and " $F_{\mathrm{s}}$ " as some "much" of one and the same set or abstraction.

Indeed the axiom scheme in set theory about abstraction can be interpreted as a scheme of tautologies, in which each name designates a set as a whole, i.e. as a "much", while the collection of elements designates as a "many" consisting of separated individuals.

The abstract and concrete objects can be considered as kinds of sets: The objects either abstract or concrete can be unified as some homogenous plurality and thus as a whole. Furthermore that whole can be considered as a new abstract object. Thus the concrete and abstract objects can be opposed as a "many" and the whole of it, or as a "many" and a "much" of one and the some quality. That intuition addresses the concept of 'set' utilized in set theory.

The quantum "principle of Hume" means properly the conservation of quantum information after decoherence ("choice") or coherence ("abstraction").

\section{Conclusions}

Any physical process is a quantum computation: Quantum computer can be equivalently represented by a quantum Turing machine. The quantum Turing machine is equivalent to Hilbert space. Quantum mechanics states that any physical state or its change is a self-adjoint operator in Hilbert space as any physical system can be considered as a quantum one. Consequently all physical process can be interpreted as the calculation of a single computer, and thus the universe being as it.

Any wave function is a value of quantum information: Any wave function can be represented as an ordered series of qubits enumerated by the positive integers. Just as an ordering of bits can represent a value of classical information, that series of qubits, equivalent to a wave function represents a value of quantum information. One can think of the qubits of the series as a special kind of digits: infinite digits As a binary digit can accept two values, that infinite digit should accept infinite number of values

All physical processes are informational: Quantum mechanics is the universal doctrine about the physical world and any physical process can be interpreted as a quantum one. Any quantum process is informational in terms of a generalized kind of information: quantum information. Consequently, all physical processes are informational in the above sense.

Quantum information is the real fundament of the world: Indeed all physical states in the world are wave functions and thus they are different values of quantum information. All physical quantities in the world are certain kind of changes of wave functions and thus of quantum information. Consequently, one can certainly state that the physical world consists only of quantum information: It is the substance of the physical world, its "matter".

Information is a bridge between two groups of fundamental philosophical concepts: The conception of information and more exactly, quantum information unifies physics and mathematics, and thus the material and the ideal world as well as the concrete and abstract objects.

The ground is the choice unifying the well-ordering of past and the uncertainness of future by the choice of present. Consequently, quantum information as the substance of the universe is the mediator between the totality and time, on the one hand, and the physical world, on the other hand.

Information can be considered also as a "bridge" between the concrete and abstract: As information is a dimensionless quantity equally well referring both to a physical entity and to a mathematical class, it can 
serve as a "bridge" between physics and mathematics and thus between the material and ideal world, between the concrete and abstract objects. In fact, quantum information being a generalized kind of information is just what allows of the physical and mathematical, the concrete and abstract to be considered as two interpretations of the underlying quantum information.

The concept of information generalized as quantum information generalizes also the concept of matter in physics as well as the corresponding quantities of matter and energy. Furthermore, quantum information can be interpreted as that generalization of information, which is applicable to infinite collections or algorithms. Thus the fundamental properties of mass or energy shared by all in the physical worlds turn out to be underlain by quantum information. The gap between the concrete objects (interpreted as physical ones) and the abstract objects is now bridged by the concept of information shared by both and underlying both kinds of objects. The quantity of information either classical (i.e. "finite") or quantum (i.e. "infinite") is defined in both cases as the amount of choices and measured in units of elementary choice: correspondingly either bits or qubits. The case of infinite choice cannot help to involve the axiom of choice and a series of counterintuitive corollaries implied by it: One of them is the so-called paradox of Skolem:

It allows of discussing the concrete and abstract objects as complementary in the sense of quantum mechanics as well as different degrees of "entanglement" between them therefore pioneering a kind of quantum epistemology as universal. The physical processes can be interpreted as informational, more exactly as quantum-informational. Any wave function determines a state of a quantum system and a state of a quantum computer defined as a quantum Turing machine, in which all bits are simply replaced by qubits infinitely many in general. Thus the concept of quantum information and calculation can unify physics and mathematics addressing some form of neo-Pythagoeranism as the common ontological ground of the concrete objects (studied by physics) and abstract ones (studied by mathematics)

\section{References:}

Aspect, Alain \& Grangier, Philippe \& Roger, Gérard (1981) "Experimental Tests of Realistic Local Theories via Bell's Theorem," Physical Review Letters 47 (7): 460-463.

Aspect, Alain \& Grangier, Philippe \& Roger, Gérard (1982) "Experimental Realization of EinsteinPodolsky-Rosen-Bohm Gedanken Experiment: A New Violation of Bell's Inequalities," Physical Review Letters 49 (2): 91-94.

Banach, Stefan and Alfred Tarski (1924) "Sur la decomposition des ensembles de points en parties respectivement congruentes," Fundamenta Mathematicae 6 (1): 244-277.

Bell, John (1964) "On the Einstein - Podolsky - Rosen paradox," Physics (New York) 1 (3): 195-200.

Boolos, George (1987) "The Consistency of Frege's Foundations of Arithmetic," in: Thomson, J. (ed.) On Beings and Sayings: Essays in Honor of Richard Cartwright, Cambridge, MA, MIT Press, pp. 3-20.

Broglie, Louis de (1925) "Recherches sur la théorie des quanta," Annales de Physique (Paris, 10-ème série) 3: 22-128.

Cantor, Georg (1897) "Beitrage zur Begrundung der transfiniten Mengenlehre (Zweiter Artikel)," Mathematische Annalen 49 (2): 207-246.

Deutsch, David (1985) "Quantum theory, the Church-Turing principle and the universal quantum computer," Proceedings of the Royal Society of London A 400: 97-117. 
Deutsch, David (1989) "Quantum computational networks," Proceedings of the Royal Society of London, Volume A 425: 73-90.

Einstein, Albert \& Podolsky, Boris \& Rosen, Nathan (1935) "Can Quantum-Mechanical Description of Physical Reality Be Considered Complete?” Physical Review 47 (10): 777-780.

Gödel, Kurt (1931) "Über formal unentscheidbare Sätze der Principia mathematica und verwandter Systeme I,“ Monatshefte der Mathematik und Physik 38 (1): 173-198.

Kochen, Simon and Specker, Ernst (1968) "The problem of hidden variables in quantum mechanics," Journal of Mathematics and Mechanics 17 (1): 59-87.

Kolmogorov, A. (1968) "Logical basis for information theory and probability theory," IEEE Transactions on Information Theory 14: 662-664.

Linnebo, Øystein (2010) "Pluralities and Sets," Journal of Philosophy 107 (3): 144-164.

Neumann, Johan von (1923) "Zur Einführung der trasfiniten Zahlen," Acta litterarum ac scientiarum Ragiae Universitatis Hungaricae Francisco-Josephinae, Sectio scientiarum mathematicarum 1 (4): 199-208.

Neumann, Johan von (1932) Mathematische Grundlagen der Quantenmechanik, Berlin, Springer, pp. 167-173 (Chapter IV.2).

Popper, Karl. (1935) Logik der forschung: zur erkenntnistheorie der modernen naturwissenschaft, Wien, Springer.

Schrödinger, Erwin (1935) "Die gegenwärtige situation in der Quantenmechanik", Die Naturwissenschaften 23 (48): 807-812; 23 (49): 823-828; 23 (50): 844-849.

Skolem, Thoralf (1922) "Einige Bemerkungen zur axiomatischen Begründung der Mengenlehre," in T. Skolem, Selected works in logic (ed. E. Fenstad), Oslo, Univforlaget (1970).

Turing, Allen (1937) "On computable numbers, with an application to the Entscheidungsproblem," Proceedings of London Mathematical Society, series 242 (1): 230-265.

Whitehead, Alfred North and Russell, Bertrand (1927) Principia Mathematica (second edition), volume 3, Cambridge, University Press.

Yao, Andrew (1993) "Quantum circuit complexity," in Proceedings of the 34th Annual Symposium on Foundations of Computer Science / November 3-5, 1993 Palo Alto, California, Los Alamitos, IEEE Computer Society. pp. 352-361

Zermelo, Ernst (1904) "Beweis, dass jede Menge wohlgeordnet werden kann," Mathematische Annalen 59 (4): 514-16.

Zermelo, Ernst (1908) "Untersuchungen über die Grundlagen der Mengenlehre I," Mathematische Annalen 65 (2): 261-281. 